\title{
Retours du colonial? Disculpation et réhabilitation de l'histoire coloniale, Catherine Coquio (dir.)
}

\section{Ilaria Vitali}

\section{(2) OpenEdition}

1 Journals

\section{Edizione digitale}

URL: http://journals.openedition.org/studifrancesi/8382

DOI: $10.4000 /$ studifrancesi.8382

ISSN: 2421-5856

\section{Editore}

Rosenberg \& Sellier

\section{Edizione cartacea}

Data di pubblicazione: 1 mai 2009

Paginazione: 213-214

ISSN: 0039-2944

\section{Notizia bibliografica digitale}

Ilaria Vitali, «Retours du colonial? Disculpation et réhabilitation de I'histoire coloniale, Catherine Coquio (dir.)», Studi Francesi [Online], 157 (LIII | I) | 2009, online dal 30 novembre 2015, consultato il 09 janvier 2021. URL: http://journals.openedition.org/studifrancesi/8382 ; DOI: https://doi.org/10.4000/ studifrancesi.8382

Questo documento è stato generato automaticamente il 9 janvier 2021.

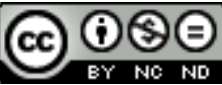

Studi Francesi è distribuita con Licenza Creative Commons Attribuzione - Non commerciale - Non opere derivate 4.0 Internazionale. 


\title{
Retours du colonial? Disculpation et réhabilitation de l'histoire coloniale, Catherine Coquio (dir.)
}

\author{
Ilaria Vitali
}

\section{NOTIZIA}

Retours du colonial? Disculpation et réhabilitation de l'histoire coloniale, Catherine CoQuio

(dir.), Nantes, L'Atalante, 2008, pp. 380.

1 Incomincia ricordando una celebre comptine coloniale l'ambizioso volume curato da Catherine Coquio - docente di Letteratura Comparata presso l'Université de Poitiers, presidente e fondatrice dell'Association internationale de recherche contre les crimes de guerre et les génocides (Aircrige) - che si propone di analizzare a vari livelli la riabilitazione del discorso coloniale, da qualche tempo sempre più presente in Francia. «Dix petits nègres s'en furent diner, / l'un d'eux but à s'en étrangler / - n'en resta plus que neuf» (p. 48). Recita così l'inizio della comptine, scritta nel 1869 e tramutata poi in canzone per insegnare ai bambini a contare educandoli sin da piccoli alla missione «civilizzatrice» europea, nonostante racconti una «gioiosa» ecatombe: dei dieci p’tits nègres iniziali non ne resta infatti, alla fine della comptine, nessuno.

2 Retours du colonial?, la provocatoria domanda retorica del titolo di questo saggio punta il dito su quello che si configura sempre più, in Francia, come un tentativo di recuperare la storia coloniale, o quantomeno, come recitava il discusso articolo 4 della legge del 23 febbraio 2005 - ora abrogato - di «enseigner de manière positive la présence de la France dans ses colonies et en outre-mer». Proprio nel momento in cui la Francia sembra volersi riconciliare con il proprio passato (si pensi alla fondazione nel 2006 del Musée du Quai-Branly e nel 2007 della Cité nationale de l'histoire de l'immigration), disculpation e réhabilitation del colonialismo sembrano essere divenuti paradossalmente obiettivi di primo piano (basti ricordare il discorso del presidente Nicolas Sarkozy a Dakar, il 26 luglio 2007). Sin dal titolo la curatrice pone dunque 
l'attenzione sugli insidiosi «ritorni» - al plurale - che si evidenziano sul piano politico, economico e culturale nell'Hexagone; ritorni che si possono distinguere prima di tutto in due assi: un ritorno critico sul «fait colonial», in prospettiva scientifica, e in qualche caso addirittura militante; un ritorno sintomatico del «fait colonial», inteso come vera e propria rivalorizzazione del discorso colonialista.

In ottica pluridisciplinare, il volume conta i contributi di diciotto autori, tra storici, scrittori, filosofi, critici letterari e psicanalisti. Le tre sezioni di cui si compone («Histoires, droits, politiques»; «Mémoires et représentations»; «Postcolonial et francophonie») affrontano nella grande diversità dei contenuti trattati i complessi problemi legati alla questione colonialismo/post-colonialismo, proponendo letture critiche approfondite e inedite. Nella prima parte, «Histoires, droits, politiques», l'attenzione è posta sul significato dell'ideologia colonialista nel periodo della postcolonie, per usare il neologismo utilizzato dallo scrittore gibutiano Abderahman Waberi, poi ripreso da Achille Mbembe nel saggio De la postcolonie (Paris, Karthala, 2000, pp. 293). Ad aprire la prima parte di questo studio è Philippe HAUSER, docente di filosofia a Nancy, con un saggio dal titolo Le mensonge comme opérateur politique (pp.65-78). Partendo dalla riflessione sulla legge del 23 febbraio 2005, considerata da Hauser come «une falsification délibérée de la réalité historique» (p.65), l'autore esamina la risorgenza di un'ideologia coloniale mutuata dal xviII secolo, che mira ad un'esaltazione lirica dei coloni, senza tener in nessun conto la situazione di estrema violenza che regnava all'epoca nelle colonie. Nell'articolo Normativité de l'état d'exception dans la période postcoloniale (pp. 79-102), lo specialista di letteratura latino-americana Gabriel PÉRIÈs, concentra invece la sua attenzione sulle implicazioni a lungo termine della legge del 1955 sull'état d'urgence, associando il postcolonialismo al fenomeno di estensione della guerra fredda nell'ambito dell'Impero francese. Nel suo contributo, Guerre coloniale française et génocide rwandais (pp.103-128), Catherine coQuio rivolge l'attenzione al genocidio del Rwanda nel 1994, mettendolo in relazione ai massacri coloniali secondo quella che definisce una sorta di continuità, instaurata dall'inizio della presenza coloniale francese prima dell'indipendenza e fino agli anni novanta. Il contributo di Sévane GARIBIAN, Qu'importe le cri pourvu qu'il y ait l'oubli (pp.129-146), prosegue la riflessione sulla discussa legge del febbraio 2005 che ha avuto anche, secondo l'autrice, il merito di scatenare il profondo malessere delle ex-colonie e d'incoraggiare e amplificare il dibattito. Questa prima sezione del volume si chiude con il virulento e polemico articolo dello scrittore senegalese Boubacar Boris Diop dall'eloquente titolo Le discours impardonnable de Nicolas Sarkozy (pp.147-156). Secondo l'autore, il discorso pronunciato dal presidente della repubblica francese a Dakar è stato apertamente colonialista e non ha fatto che insistere su una serie di «désolants clichés de l'ethnologie coloniale du XIX ${ }^{\mathrm{e}}$ siècle» (p. 148).

4 Membro del gruppo di ricerca «Anthropologie des représentations du corps» al CNRS di Marsiglia, lo storico Pascal Blanchard inaugura la seconda parte del volume, «Mémoires et représentations», con un articolo che riprende il dibattito sulla legge del 23 febbraio 2005, dal titolo L'impossible débat colonial (pp. 159-176), in cui evidenzia la pericolosità dei metodi pedagogici che mirano all'esaltazione del discorso coloniale a livello scolastico. È poi la volta della psichiatra Alice Cherki che, nell'articolo Confiscations des mémoires et empêchement des identifications plurielles (pp. 177-186), mette in relazione, tra l'altro, la privazione dei diritti dei colonizzati con le recenti rivolte nelle banlieue dei figli degli immigrati. Segue poi un intervento che si concentra più da vicino sullo spazio 
letterario: quello dello specialista Bernard Mouralis, professore emerito dell'Université Cergy-Pontoise, dal titolo La colonisation chez des écrivains africains depuis 1990 (pp. 187-210), che analizza diversi autori-chiave della letteratura africana (Ampâté Bâ, Tierno Monénembo, Ahmadou Kourouma, Mongo Beti). Nello studio Reconstruction et déconstruction de soi: pour une "communication" entre deux mondes (pp. 211-220), Nils ANDERSSON, fondatore delle edizioni La Cité (Losanna), s'interroga invece sulla possibilità di costruire il sé sotto lo sguardo del colonizzatore, facendo riferimento soprattutto all'esperienza algerina, di cui è stato protagonista. La scrittrice Zahia RAHMANI si concentra sulla problematica e ancora oggi discussa figura dell'harki partendo dall'esperienza vissuta in prima persona da suo padre, nell'articolo Le harki comme spectre ou l'écriture du déterrement (pp. 221-238). Chiude questa seconda parte del volume l'articolo di Alain DENEAULT Les symboles coloniaux au service de l'humour noir 'offshore' (pp. 239-253), in cui l'autore crea un'inedita relazione/riflessione sul concetto di «paradiso fiscale» e di «île lointaine».

5 La terza e ultima parte del volume, «Postcolonial et francophonie», si divide a sua volta in due sottosezioni: la prima si rivolge più in generale al concetto di «postcoloniale», mentre la seconda, «Francophonie: lire, écrire, éditer» si concentra in modo specifico sugli aspetti letterari e editoriali dell'universo francofono. I primi tre interventi sono dedicati agli aspetti più teorici, partendo dalla figura simbolo di Edward Said, iniziatore di quelli che diventeranno i postcolonial studies, analizzati nei contributi di Marc NICHANIAN (pp. 259-276) e di Françoise VERGES (pp. 277-288), fino a sottolineare il «ritardo» della critica francese al riguardo, nel contributo di Tiphaine SAMOYAULT (pp. 289-299), dall'eloquente titolo Les réticences française à l'égard des études postcoloniales. I quattro studi che compongono l'ultima sottosezione analizzano infine le «conseguenze» letterarie del colonialismo/postcolonialismo. L'intervento di Anthony MANGEON (pp.303-330), si sofferma sull'Africa di Yambo Ouologuem, Ahmadou Kourouma e Achille Mbembe mentre Laure CORET (pp.331-346) si concentra sulla letteratura femminile antillana nel saggio Ecriture postcoloniale, écriture de soi. Chiudono il volume due contributi sul concetto di «francofonia» e di «scrittore francofono» (con particolare riferimento all'area africana): quello di Elö̈se BREZAULT (pp. 347-358), che rivolge la sua attenzione al dibattito ancora vivo tra littérature francophone e littérature franco-française e quello dell'artista del Ciad Koulsy LAмко (pp. 359-369), che si propone di mostrare, come recita il titolo del suo studio, Leurres et lueurs de la francophonie.

Un volume prezioso, che non cade nel facile errore di opporre al dogma della réhabilitation du discours colonial altri dogmi, ma anzi, argomenta, analizza, sonda, arrivando ad una vera e propria decostruzione dell'ideologia colonialista nel periodo della «postcolonia». Un saggio di estremo interesse - anche per il ricchissimo apparato bibliografico - per chi si occupa di francofonia e, più in generale, di studi postcoloniali. 